Article

\title{
Focus on Clozapine Withdrawal- and Misuse-Related Cases as Reported to the European Medicines Agency (EMA) Pharmacovigilance Database
}

\author{
Stefania Chiappini ${ }^{1}{ }^{\circledR}$, Fabrizio Schifano ${ }^{1, *}$, John Martin Corkery ${ }^{1}\left[\right.$ and Amira Guirguis ${ }^{2} \mathbb{C}$ \\ 1 Psychopharmacology, Drug Misuse, and Novel Psychoactive Substances Research Unit, School of Life and \\ Medical Sciences, University of Hertfordshire, Hatfield, Hertfordshire AL10 9AB, UK; \\ stefaniachiappini9@gmail.com (S.C.); j.corkery@herts.ac.uk (J.M.C.) \\ 2 Swansea University Medical School, Institute of Life Sciences 2, Swansea University, Singleton Park, \\ Swansea SA2 8PP, UK; amira.guirguis@swansea.ac.uk \\ * Correspondence: f.schifano@herts.ac.uk
}

Received: 14 January 2020; Accepted: 14 February 2020; Published: 16 February 2020

\begin{abstract}
Background: Clozapine is of high clinical relevance for the management of both treatment-resistant schizophrenia and psychotic disturbances with concurrent drug misuse. Although the molecule presents with a range of well-known side-effects, its discontinuation/withdrawal syndrome has been only anecdotally described. Aims: the 2005-2018 European Medicines Agency (EMA) dataset of Adverse Drug Reactions (ADRs) was analyzed to identify and describe possible clozapine withdrawal- and misuse-/abuse-/dependence-related issues. Method: A descriptive analysis of clozapine-related ADRs was performed when available, data on ADRs' outcome, dosage, and possible concomitant drug(s) were considered. Results: Out of 11,847 clozapine-related ADRs, some $599(5.05 \%)$ were related to misuse/abuse/dependence/withdrawal issues, including 258 withdrawal-related (43.1\%); 241 abuse-related (40.2\%); and 80 intentional product misuse-related $(13.3 \%)$ ADRs. A small number of overdose- and suicide-related ADRs were reported as well. Clozapine was typically (69.2\%) identified alone, and most (84.7\%) fatalities/high-dosage intake instances were reported in association with a history of substance abuse. Conclusions: Previous suggestions about the possibility of a clozapine discontinuation/withdrawal occurrence are here supported, but further studies are needed. However, the misuse/abuse cases here identified might be difficult to interpret, given the lack of studies highlighting the possible recreational use of clozapine. The high-dosage intake, fatal outcomes and clozapine/polydrug abuse issues reported here may, however, be a reason for concern.
\end{abstract}

Keywords: clozapine; adverse drug reactions; misuse; withdrawal; dependence; overdose

\section{Introduction}

Clozapine was first synthesized in 1959 [1] and made available on the European market in 1971 for the treatment of schizophrenia [2]. If compared with remaining first-generation antipsychotic agents, clozapine is characterized by a lack of extrapyramidal side-effects [2]. After having been withdrawn from the European market in 1976 due to fatal cases of agranulocytosis, clozapine was approved by the Food and Drug Administration (FDA) in 1989 for treatment-resistant schizophrenia [2,3]. It was then approved in the USA in 2002 for reducing recurrent suicidal behavior in patients with schizophrenia or schizoaffective disorder. Other unlicensed uses include treatment-resistant bipolar disorder, aggression in patients with psychosis, and other brain disorders unresponsive to remaining treatments [4]. 


\subsection{Pharmacodynamic Considerations}

Clozapine is a dibenzodiazepine designated as a Multi-Acting Receptor-Targeted Antipsychotic (MARTA), deriving its therapeutic effects from its action across various neurotransmitter systems $[4,5]$. In fact, a combination of pharmacological effects is unique to clozapine: (1) although the molecule presents with affinity levels for a range of dopamine/DA receptors (e.g., D1, D2, D3, D4, and D5; [6]), the blockade of dopamine 2 and 4 receptors is particularly relevant, with a preferential affinity for D4 over D2 receptors; this blockade seems to be effective in reducing positive symptoms of psychosis and stabilizing affective symptoms [4]; (2) 5-hydroxytryptamine/5-HT 2A receptor antagonism, causing enhancement of DA release in certain brain regions, and thus reducing motor side-effects and possibly improving cognitive and affective symptoms associated with schizophrenia [4]. Conversely, the clinical effects of the reported interactions at 5HT2C; 5HT1A; 5-HT6; and 5-HT7 receptors [6] might be less clear; (3) $\alpha 2 \mathrm{C}$-adrenergic receptor blockade; this may contribute to the clozapine-related improvement of cognitive function [7]; (4) significant antimuscarinic and antihistaminergic H1 effects, which may well contribute to the central effect [8]; and (5) possible modulatory actions on a dysfunctional glutamatergic system, improving schizophrenia symptoms and contrasting illness progression [1,4,9-11]. More precisely, clozapine interacts with the N-methyl-D-aspartate (NMDA) receptor complex, affecting either the glycine site of the NMDA receptor or tentatively inhibiting the glycine transporter [12]. Moreover, both clozapine and its major metabolite N-desmethylclozapine behave as: (i) delta-opioid receptor agonists [13-17]; (ii) cannabinoid CB1 receptor agonists [18]; and (iii) antagonists at muscarinic receptors [8]. Indeed, these i-iii pharmacological activities are, per se, typically associated with the occurrence of pleasurable effects [19-22], which could suggest a theoretical potential for clozapine to be misused by vulnerable individuals. Finally, further pharmacological clozapine activities which should be better understood in terms of their clinical effects may include both interaction with GABA-B receptors [22]; and antagonistic actions at D (2)/D (3)/D (4) receptors [23].

The therapeutic response depends on plasma clozapine concentrations, which may be influenced by many factors such as age, gender, and smoking [5], and should normally reach $350 \mathrm{ng} / \mathrm{mL}$ [4,5]; levels greater than $700 \mathrm{ng} / \mathrm{mL}$ are often not well tolerated [4]. Clozapine is metabolized by CYP450 enzymes, specifically by CYP450 1A2, 2D6 and 3A4 enzymes [5], and its metabolism may be influenced by CYP450 1A2 inhibitors, such as fluvoxamine and ciprofloxacin [14]. Remaining interactions may occur with both strong CYP450 2D6 inhibitors (e.g., bupropion, duloxetine, paroxetine, fluoxetine) and strong CYP450 3A4 (e.g., ketoconazole) inhibitors [4,15].

\subsection{Clozapine Abuse Issues and Substance Use Disorders}

Substance use disorders (SUDs), typically involving alcohol, cannabis, and cocaine, commonly occur in patients with schizophrenia, supposedly due to epidemiological and genetic determinants of risk for both psychosis and addiction [1,16]. This co-occurrence ('dual diagnosis') has a negative effect on the course of schizophrenia, due to increased rates of hospitalization, decreased compliance with medication, increased violence and suicide, general deterioration of the patients' condition, and overall increased societal costs $[1,17]$. In particular, the positive symptoms of schizophrenia are generally exacerbated by the intake of stimulant drugs, such as cocaine, amphetamine derivatives [24], and synthetic cathinones [25]. Due to clozapine's effectiveness, there has been support for considering the molecule in limiting substance use in patients with schizophrenia [26], to achieve both a reduction in substance use [27,28] and an improvement in positive/negative schizophrenia symptoms [29]. Additionally, differently from other antipsychotics, such as quetiapine, which has showed in recent years to be a strong potential for misuse and abuse [30-33], the recreational use of clozapine has not been noted in the literature. Conversely, clozapine withdrawal is a phenomenon which has already been described, even at therapeutic dosages [9]. However, the risk of withdrawal may arguably be significant only when a psychoactive molecule is being misused/ingested at higher dosages. Since SUD patients may be vulnerable to misuse prescribed medications, one could argue that it is relevant to identify and assess any possible clozapine misuse/abuse/withdrawal and dependence issues. 


\subsection{Aims}

We aimed here at identifying and describing the number of European Medicines Agency (EMA) database cases of misuse, abuse, dependence, and withdrawal specifically relating to clozapine; suicide-related cases and fatalities were also considered.

\section{Methods}

\subsection{Data Source and Acquisition}

EudraVigilance (EV) manages and analyzes information on suspected Adverse Drug Reactions (ADRs) to medicines that have been authorized in the European Economic Area (EEA), according to Directive 2001/83/EC and Regulation (EC) No 726/2004 [34]. In order to investigate the aspects above, EV was specifically requested to provide all Level 2A data [34] comprising case reports of clozapine-related misuse, abuse, dependence, and withdrawal ADRs, which were obtained in the form of line listings, relating to the 2005-June 2018 time-frame. Differently from the publicly available data from the EV website, Level 2A data were presented as Excel sheets divided into information sections reporting in a standardized format according to the Medical Dictionary for Regulatory Activities (MedDRA), which is the internationally agreed list of terms that supports the coding of ADRs [35], which are identified through Preferred Terms (PT). Indeed, MedDRA is a comprehensive and highly standardized medical terminology developed by the International Council for Harmonization of Technical Requirements for Pharmaceuticals for Human Use (ICH) (https://www.meddra.org/) in the late 1990s to facilitate the international sharing of regulatory information for medical products. Such listings showed all information related to the ADR, the patient, the drug, the reporter, and the diagnosis. In line with previous studies from our group [36-39], the ADRs considered here were, per se, voluntary and unsolicited communications [34] reported by both Regulatory Authorities of the EU Member States where the reaction occurred and/or by the Marketing Authorization Holders for those ADRs occurring outside the EEA. Individual Case Safety Reports, such as voluntary reports, refer to the format and content for the reporting of one or several suspected ADRs in relation to a medicinal product that occur in a single patient at a specific point of time [40]. 'Suspect' ADRs were selected, meaning clozapine was considered as 'suspected' for the reaction reported [34]. Within the standardized MedDRA Query (SMQ) 'drug abuse, dependence and withdrawal' section, we identified the following ADRs: dependence, drug abuse, drug abuser, drug dependence, drug diversion, drug withdrawal convulsions, drug withdrawal syndrome, drug withdrawal neonatal syndrome, intentional product misuse, product use issue, substance abuse, and withdrawal syndrome. For the definition of 'abuse'; 'addiction'; 'dependence'; and 'misuse' please refer to references [35-40]. Moreover, in accordance with MedDRA, 'withdrawal' is identified in association with the abrupt cessation or reduction in intake of a drug in a habituated person. A substance-specific syndrome may follow, with withdrawal symptoms varying according to the psychoactive substance used and generally opposite the acute effects of drug. These include nonspecific symptoms e.g., nausea, diarrhea or constipation, profuse sweating, increase in respiratory rate, and tachycardia; remaining common symptoms include anxiety, restlessness, irritability, insomnia, and impaired attention [35].

\subsection{Data Analysis}

Each case report may refer to one or more reporter; one or more ADR(s); as well as to one or more medicinal product(s). Therefore, a case may be represented by more than one row in the other line listings. All rows of the case have the same 'EV Local Report Number', unequivocally identifying an individual case. Thus, the number of suspected ADRs can be different from the number of case reports as one case report may refer to several suspected ADRs. Moreover, the number of patients can be different from the number of case reports as a patient may have been described in more than one case. Finally, ADRs' numbers differed from those referring to case reports/single patients since different reporters/senders could have independently flagged the same ADR to the EMA. 
Patients' data were analyzed using a range of parameters, including: socio-demographic characteristics (age and gender); source/reporter country (EEA or non-EEA) and reporter qualification (i.e., pharmacist, physician); outcomes (fatal, recovered, resolved); clozapine dosage; possible concomitant drug(s); and diagnosis/reporter's comments, if recorded [37]. The analysis included cases of overdoses, suicides, and fatalities.

Suicidal behavior was here defined as 'completed suicide', 'intentional self-injury', 'suicidal behavior', 'suicidal ideation', 'suicide attempt', 'self-injurious ideation', and 'intentional self-injury.' 'Overdose', including 'intentional overdose', was not necessarily interpreted as being a suicidal attempt [35]. The analysis included here both a descriptive study of the dataset and the Confidence Interval (CI) values [41].

\subsection{Ethics' Issues}

Complying with applicable Personal Data Protection legislation (Regulation (EC) No 45/2001 and Regulation (EC) No 1049/2001 on the protection of privacy and integrity of individuals, certain data elements, including names/identifiers of individuals involved or country-specific information were not disclosed by the EMA to safeguard the identity of individuals [42]. The study was ethically approved in March 2018 by the University of Hertfordshire Ethics' Committee, with reference number LMS/PGR/UH/03234.

\section{Results}

The EMA dataset included a total of 13,596 clozapine-related ADRs. A total of 11,847 ADRs were considered as 'suspect', i.e., as suspected to be related with clozapine. Of those, 599/11,847 (5.05\% CI 95\% 597-601) related to misuse/abuse/dependence/withdrawal ADRs and were associated with 559 unique subjects. These included: 258 ADRs (258/599 $=43.1 \%$ CI 95\% 256-260) relating to 'withdrawal syndrome' issues; 241 abuse-related ADRs (241/599 = 40.2\% CI 95\% 239-242); and 80 ADRs $(80 / 599=13.3 \%$ CI 95\% 76-84) relating to 'intentional product misuse.' A small number of overdose- $(n=29)$ and suicidal behavior-related ADRs $(n=29)$ were reported here, resulting in death, respectively, in two and 11 cases (Table 1 ).

Table 1. Analysis of the EudraVigilance (EV) clozapine-related misuse/abuse/dependence and withdrawal ADRs (2005-June 2018).

\begin{tabular}{cc}
\hline $\begin{array}{c}\text { EudraVigilance (EV) Clozapine-Related Misuse/Abuse/Dependence and } \\
\text { Withdrawal ADRs (2005-June 2018) }\end{array}$ & $n$ \\
\hline Total "suspect”' clozapine-related ADRs & 11,847 \\
Clozapine-related 'abuse, dependence and withdrawal' ADRs & $599(C I 95 \% 595-603)$ \\
Drug abuse & $n$ individual cases = 559) \\
Drug abuser & 198 \\
Substance abuse & 1 \\
Dependence & 42 \\
Drug dependence & 7 \\
Drug diversion & 6 \\
Intentional product misuse & 1 \\
Product use issue & 80 \\
Drug withdrawal convulsions & 4 \\
Drug withdrawal neonatal syndrome & 1 \\
Drug withdrawal syndrome & 1 \\
Withdrawal syndrome & 91 \\
Intentional overdose & 165 \\
Orverdose & 12 \\
\hline
\end{tabular}


Table 1. Cont.

\begin{tabular}{cc}
\hline $\begin{array}{c}\text { EudraVigilance (EV) Clozapine-Related Misuse/Abuse/Dependence and } \\
\text { Withdrawal ADRs (2005-June 2018) }\end{array}$ & $n$ \\
\hline Completed suicide & 9 \\
Intentional self-injury & 4 \\
Suicidal behavior & 1 \\
Suicidal ideation & 4 \\
Suicide attempt & 7 \\
Self-injurious ideation & 4 \\
\hline
\end{tabular}

Patients were typically males $(379 / 559=67.8 \%$ CI 95\% 378-380), in the 18-65 years age range. Most typical senders were pharmaceutical companies (303/559 $=54.2 \%$ CI 95\% 301.5-304.5) and regulatory authorities (241/559 = 43.11\% CI 95\% 239-243) (Table 2).

Table 2. Description of clozapine-related misuse, abuse, dependence and withdrawal cases. F: female; M: male; N: no; Y: yes.

\begin{tabular}{|c|c|}
\hline $\begin{array}{l}\text { Clozapine-Related Misuse, Abuse, } \\
\text { Dependence and Withdrawal Cases }\end{array}$ & $n$ of Unique Patients \\
\hline & 559 \\
\hline Age range (years) & $\begin{array}{c}3 \text { neonates, } \\
1 \text { child ( } 5 \text { years), } \\
2 \text { adolescents (15-16 years), } \\
78 \text { adults ( } 18-65 \text { years), } \\
1 \text { elderly ( } 67 \text { years), } \\
474 \text { Not specified }\end{array}$ \\
\hline Gender & $\begin{array}{c}171 \mathrm{~F}, \\
379 \mathrm{M}, \\
9 \text { Not Specified }\end{array}$ \\
\hline Sender & $\begin{array}{l}\text { 241 Regulatory authority, } \\
\text { 303 Pharmaceutical company, } \\
\text { 9 Other (distributor, study sponsor, contract research organization), } \\
6 \text { Not Specified }\end{array}$ \\
\hline \multicolumn{2}{|l|}{ Outcome } \\
\hline Resulted in death & 46 Y, 448 N, 65 Not Specified \\
\hline Life threatening & 35 Y, 447 N, 77 Not Specified \\
\hline Required a prolonged hospitalization & 298 Y, 219 N, 42 Not Specified \\
\hline Disabling & $8 \mathrm{Y}, 467$ N, 84 Not Specified \\
\hline
\end{tabular}

The number of cases increased year by year, with a peak in 2008 (120/559 cases) (Figure 1). 


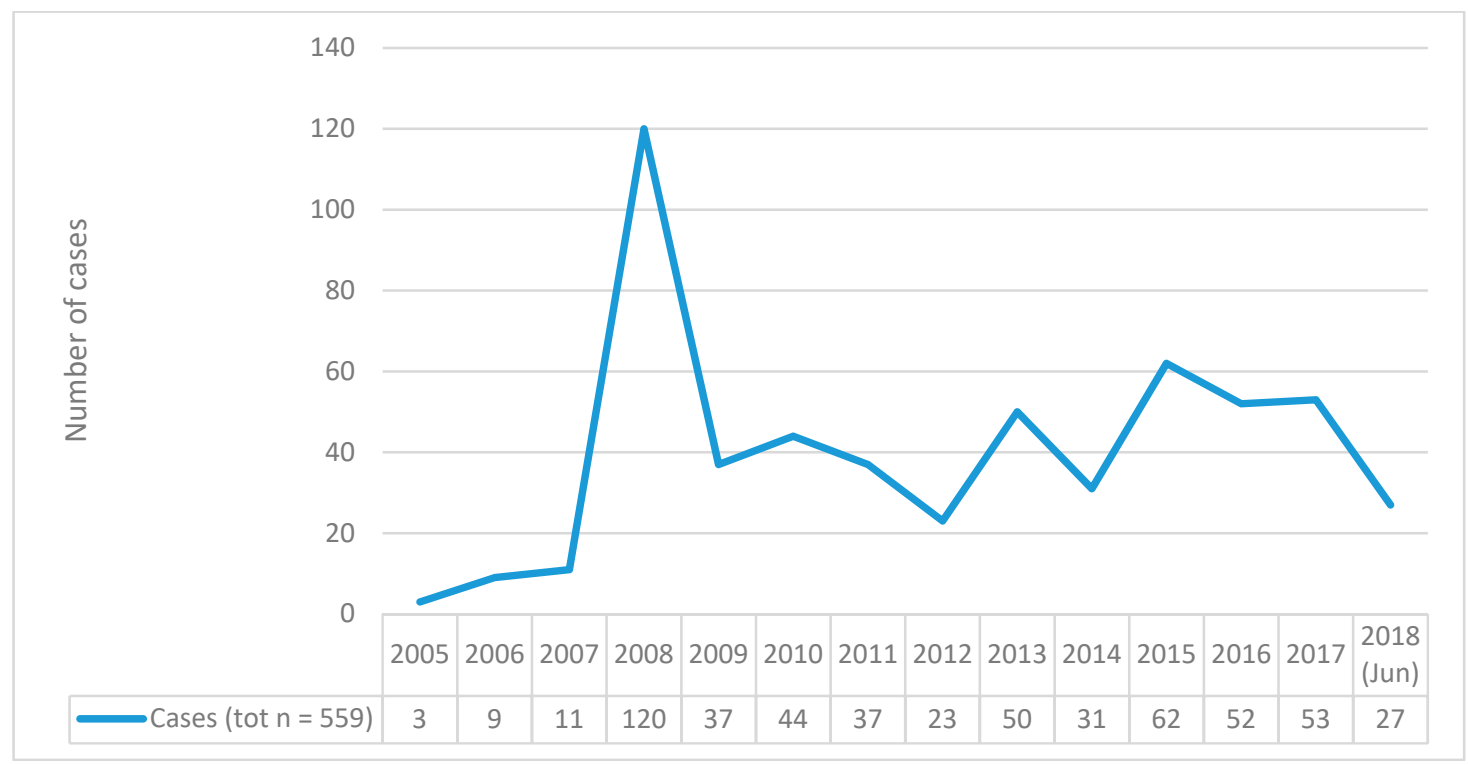

Figure 1. Total Number of clozapine-related misuse, abuse, dependence, withdrawal cases reported to EudraVigilance (EV) per year (2005-June 2018).

Oral intake occurred here in 533/559 cases (95.3\% CI 95\% 532.5-533.5); when recorded, clozapine dosages varied from $12.5 \mathrm{mg} /$ day to high/unlicensed levels (i.e., $2800-5600 \mathrm{mg} /$ day; Figure 2). Only a few cases $(n=7)$, however, reported high (e.g., $>1000 \mathrm{mg}$ ) levels. When the relating clinical data were made available, these cases were typically described as 'intentional self-injury', 'completed suicide', and 'drug abuse').

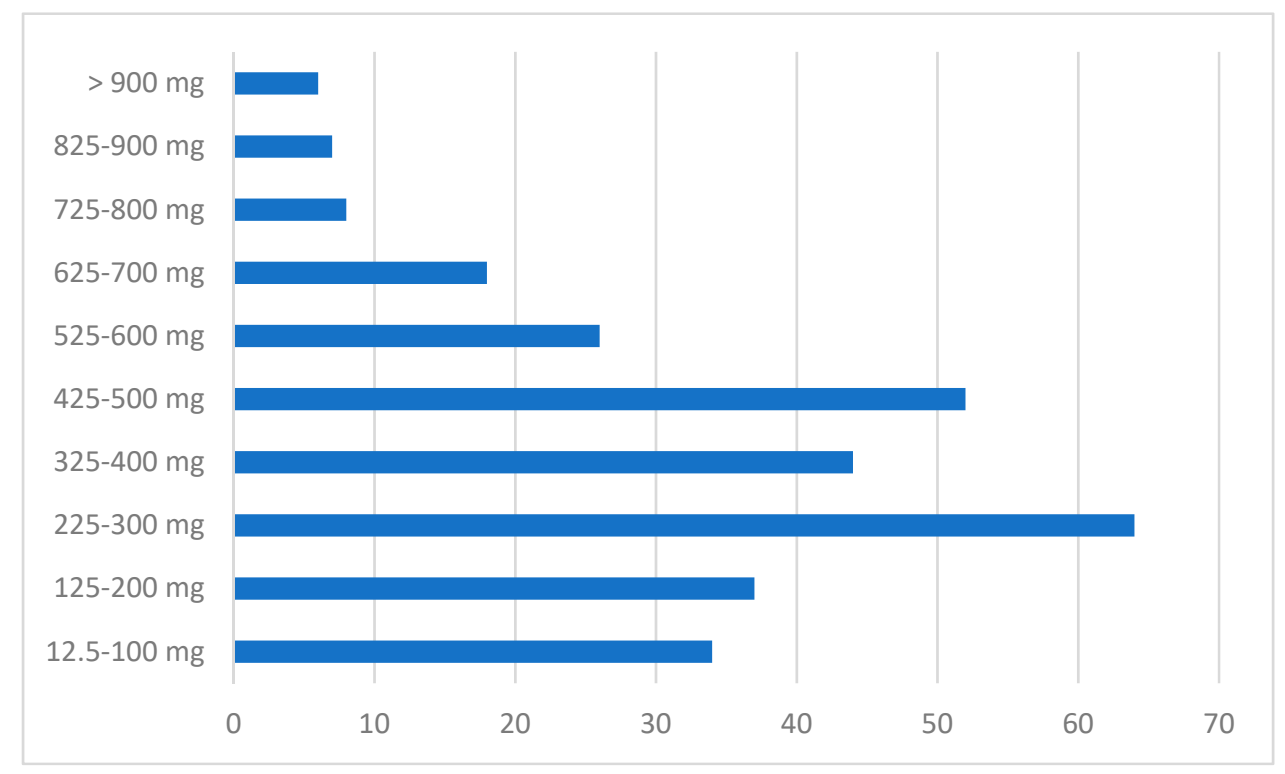

Figure 2. Dosages of clozapine reported to the EudraVigilance (EV) dataset of clozapine-related misuse, abuse, dependence, withdrawal ADRs.

Clozapine was the sole molecule reported in 387/559 (69.2\% of cases; CI 95\% 386-388); remaining drugs included: first/second generation antipsychotics (55/559 cases; $9.8 \%$ ); benzodiazepines (clonazepam, diazepam, and lorazepam; 54/559 cases; 9.7\%); antidepressants (citalopram, escitalopram, sertraline, and venlafaxine; 33/559 cases; 5.9\%); and mood stabilizers (valproic acid, carbamazepine, lamotrigine, lithium, and gabapentin; 20/559 cases; $3.6 \%$ ). Illicit drugs most typically reported here 
were opioids (15/559 cases), amphetamines (10/559), cannabis, and alcohol (each in seven cases). In 30 cases, two or more prescribed/recreational drugs were recorded in association with clozapine (Table 3).

Table 3. Clozapine alone or in combination rates of identification, as recorded by the EudraVigilance

(EV) dataset of misuse, abuse, dependence, and withdrawal cases.

\begin{tabular}{cc}
\hline $\begin{array}{c}\text { Clozapine-Related Misuse, Abuse, Dependence and Withdrawal } \\
\text { Cases Recorded by the EudraVigilance (EV) Dataset }\end{array}$ & Tot $(\boldsymbol{n}=\mathbf{5 5 9})$ \\
\hline $\begin{array}{c}\text { Clozapine ingested as a lone drug } \\
\text { Clozapine identified in combination with remaining drugs }\end{array}$ & $387(69.2 \%)$ \\
\hline Prescribing drugs & $172(30.7 \%)$ \\
Other antipsychotics & 55 \\
Benzodiazepines & 54 \\
Antidepressants & 33 \\
Mood stabilizers & 20 \\
Z-drugs & 4 \\
Recreational drugs & 15 \\
Opioids & 10 \\
Amphetamine derivatives & 7 \\
Cannabis & 7 \\
Alcohol & 4 \\
Cocaine & 1 \\
\hline
\end{tabular}

The ADRs required in most cases a prolonged hospitalization (298/559 $=53.3 \%$ CI 95\% 296.5-299.5), while some 46 cases $(46 / 559=8.2 \%)$ resulted in death (Tables 2 and 4$)$. Of these 46 clozapine-related fatalities, $39(84.7 \%)$ were associated with a previous history of substance abuse (Table 4 ).

Table 4. Description of the clozapine-related fatalities' cases reported to the EudraVigilance (EV) dataset (2005-June 2018). F: female; M: male.

\begin{tabular}{|c|c|c|c|c|c|}
\hline & & $\begin{array}{l}\text { Clozapine } \\
\text { Dosage (mg) }\end{array}$ & $\begin{array}{l}\text { Concomitant } \\
\text { Drugs }\end{array}$ & Medical History & $\begin{array}{l}\text { Reactions according to the MedDRA } \\
\text { Dictionary (Preferred Terms-PT) }\end{array}$ \\
\hline 1 & $\begin{array}{l}\text { M, Adult (30 } \\
\text { yy) }\end{array}$ & $200 \mathrm{mg}$ & $\begin{array}{l}\text { Benzodiazepines } \\
\text { Alcohol }\end{array}$ & & $\begin{array}{c}\text { Intentional product misuse; } \\
\text { Antipsychotic drug level increased } \\
\text { (clozapine plasmatic } 1.1 \mathrm{mg} / \mathrm{mL} \text { ); Blood } \\
\text { pressure decreased; Loss of consciousness; } \\
\text { Poisoning; Dysarthria; Loss of } \\
\text { consciousness; Blood alcohol increased }\end{array}$ \\
\hline 2 & $\begin{array}{l}\text { M, Adult }(48 \\
\text { yy) }\end{array}$ & N/A & $\begin{array}{l}\text { Cocaine } \\
\text { Opioids } \\
\text { Z-drugs }\end{array}$ & $\begin{array}{l}\text { Hypertension; } \\
\text { Schizophrenia; } \\
\text { Chronic obstructive } \\
\text { pulmonary disease; } \\
\text { Arteriosclerosis }\end{array}$ & $\begin{array}{c}\text { Substance abuse; Toxicity to various } \\
\text { agents }\end{array}$ \\
\hline 3 & $\begin{array}{l}\text { M, Adult }(25 \\
\text { yy) }\end{array}$ & $300 \mathrm{mg}$ & $\begin{array}{l}\text { Benzodiazepines } \\
\text { Opioids }\end{array}$ & & $\begin{array}{c}\text { Cardiac arrest; Arrhythmia } \\
\text { Toxicity to various agents (methadone } \\
\text { and clozapine); } \\
\text { Drug abuse; Drug level increased } \\
\text { (methadone and clozapine) }\end{array}$ \\
\hline 4 & $\begin{array}{l}\text { M, Adult }(44 \\
\text { yy) }\end{array}$ & N/A & Opioids & $\begin{array}{c}\text { Hypertension; } \\
\text { Alcoholism; } \\
\text { Depression; Epigastric } \\
\text { discomfort; Anxiety }\end{array}$ & $\begin{array}{l}\text { Pulmonary embolism; } \\
\text { Drug abuse }\end{array}$ \\
\hline 5 & M, Adult & $400 \mathrm{mg}$ & Alcohol & & $\begin{array}{c}\text { Liver function test abnormal; } \\
\text { Blood alkaline phosphatase increased; } \\
\text { Gamma-glutamyl transferase increased; } \\
\text { Asphyxia; Alcohol abuse; } \\
\text { Drug abuse (heroin) }\end{array}$ \\
\hline 6 & M, Adult (24yy) & N/A & Alcohol & Anemia & Drug abuse (Clozaril abuse) \\
\hline
\end{tabular}


Table 4. Cont.

\begin{tabular}{|c|c|c|c|c|c|}
\hline & & $\begin{array}{c}\text { Clozapine } \\
\text { Dosage (mg) }\end{array}$ & $\begin{array}{l}\text { Concomitant } \\
\text { Drugs }\end{array}$ & Medical History & $\begin{array}{l}\text { Reactions according to the MedDRA } \\
\text { Dictionary (Preferred Terms-PT) }\end{array}$ \\
\hline 7 & $\mathrm{~F}$ & $300 \mathrm{mg}$ & & $\begin{array}{l}\text { Alcoholism; Mental } \\
\text { impairment; } \\
\text { Depression; Treatment } \\
\text { noncompliance; } \\
\text { Deep vein thrombosis }\end{array}$ & $\begin{array}{l}\text { Death; Withdrawal syndrome; } \\
\text { Acute psychosis; Cognitive disorder; } \\
\text { Amnesia; Speech disorder; Gait } \\
\text { disturbance; Chills }\end{array}$ \\
\hline 8 & F, Adult (54yy) & $350 \mathrm{mg}$ & Benzodiazepines & & $\begin{array}{l}\text { Withdrawal syndrome; Insomnia; } \\
\text { Completed suicide }\end{array}$ \\
\hline 9 & $\begin{array}{l}\text { M, Adult (66 } \\
\text { yy) }\end{array}$ & N/A & & & Completed suicide; Drug abuse \\
\hline 10 & F, Adult (25yy) & N/A & Antidepressants & & Completed suicide; Drug abuse \\
\hline 11. & M, Adult (31yy) & N/A & & & Completed suicide; Drug abuse \\
\hline 12 & M & N/A & & $\begin{array}{l}\text { Obesity; } \\
\text { Gastroesophageal } \\
\text { reflux; Drug abuse }\end{array}$ & Drug abuse; Death \\
\hline 13 & $\mathrm{~F}$ & N/A & $\begin{array}{l}\text { Cocaine } \\
\text { Benzodiazepines } \\
\text { Opioids }\end{array}$ & Drug dependence & $\begin{array}{c}\text { Drug abuse (cocaine, leponex and } \\
\text { probably benzodiazepines); Death; } \\
\text { Hyperthermia malignant; } \\
\text { Cardiac arrest; Circulatory collapse; } \\
\text { Delirium }\end{array}$ \\
\hline 14 & M & N/A & & Hepatitis & $\begin{array}{c}\text { Drug abuse (possible use of } \\
\text { non-prescribed drugs); Death }\end{array}$ \\
\hline 15 & M & N/A & & $\begin{array}{c}\text { Salivary } \\
\text { hypersecretion; } \\
\text { Drug abuse (crack } \\
\text { cocaine, marijuana, } \\
\text { alcohol); } \\
\text { Extrapyramidal } \\
\text { disorder }\end{array}$ & $\begin{array}{c}\text { Nonspecific reaction; Treatment } \\
\text { noncompliance; } \\
\text { Drug abuse (mixed polysubstance abuse); } \\
\text { Death }\end{array}$ \\
\hline 16 & $\mathrm{~F}$ & N/A & $\begin{array}{c}\text { Mood } \\
\text { stabilizers } \\
\text { Antipsychotics }\end{array}$ & & Drug abuse \\
\hline 17 & M & $1000 \mathrm{mg}$ & & $\begin{array}{l}\text { Hypercholesterolemia; } \\
\text { Substance abuse; } \\
\text { Hypertension; } \\
\text { Schizophrenia; } \\
\text { Gastro-esophageal } \\
\text { reflux disease }\end{array}$ & $\begin{array}{c}\text { Hematemesis; Drug abuse; } \\
\text { Cardiac arrest; Completed suicide; } \\
\text { Toxicity to various agents; Intentional } \\
\text { overdose; } \\
\text { Resuscitation; Seizure; } \\
\text { Tachycardia }\end{array}$ \\
\hline 18 & F, Adult (27yy) & N/A & & & Drug abuse \\
\hline 19 & M, Adult (30yy) & N/A & & & $\begin{array}{l}\text { Myocardial infarction; Drug abuse; Eye } \\
\text { swelling; Glossodynia; Tongue coated; } \\
\text { Swollen tongue }\end{array}$ \\
\hline 20 & $\mathrm{~F}$ & N/A & & & $\begin{array}{c}\text { Intentional self-injury; Meningioma; } \\
\text { Drug abuse; } \\
\text { Hypoglycemia; Peripheral venous } \\
\text { disease; Hepatic steatosis; Scar; } \\
\text { Pneumonia; } \\
\text { Bronchitis; Arteriosclerosis coronary } \\
\text { artery; Purulent discharge; Pulmonary } \\
\text { congestion; Death }\end{array}$ \\
\hline 21 & M, Adult (26yy) & $800 \mathrm{mg}$ & & & $\begin{array}{c}\text { Coronary artery disease; Drug abuse; } \\
\text { Hallucination, auditory; Epilepsy; } \\
\text { Depressed mood }\end{array}$ \\
\hline 22 & M, Adult (31yy) & N/A & & & Death; Substance abuse \\
\hline 23 & M & N/A & & $\begin{array}{l}\text { Surgery; Intentional } \\
\text { self-injury; Bipolar } \\
\text { disorder }\end{array}$ & $\begin{array}{l}\text { Agitation; Death; Drug abuse; } \\
\text { Suicidal ideation; Nasal septum deviation }\end{array}$ \\
\hline 24 & M, Adult (30yy) & N/A & & & $\begin{array}{c}\text { Autonomic nervous system imbalance; } \\
\text { Catatonia; Drug withdrawal syndrome; } \\
\text { Dyskinesia; Neuroleptic malignant } \\
\text { syndrome; } \\
\text { Psychotic disorder }\end{array}$ \\
\hline
\end{tabular}


Table 4. Cont.

\begin{tabular}{|c|c|c|c|c|c|}
\hline & & $\begin{array}{c}\text { Clozapine } \\
\text { Dosage (mg) }\end{array}$ & $\begin{array}{l}\text { Concomitant } \\
\text { Drugs }\end{array}$ & Medical History & $\begin{array}{l}\text { Reactions according to the MedDRA } \\
\text { Dictionary (Preferred Terms-PT) }\end{array}$ \\
\hline 25 & M, Adult (36yy) & N/A & & & $\begin{array}{l}\text { Alcohol abuse; Drug abuse; } \\
\text { Myocardial infarction; Coronary artery } \\
\text { disease }\end{array}$ \\
\hline 26 & M & $200 \mathrm{mg}$ & & & Drug abuse; Obesity \\
\hline 27 & M, Adult (34yy) & $350 \mathrm{mg}$ & & & $\begin{array}{c}\text { Circulatory collapse; Intentional product } \\
\text { misuse; } \\
\text { Cardiopulmonary failure }\end{array}$ \\
\hline 28 & M, Adult (33yy) & N/A & $\begin{array}{l}\text { Benzodiazepines } \\
\text { Opioids }\end{array}$ & & Death; Drug abuse (heroin, temazepam) \\
\hline 29 & & N/A & & $\begin{array}{l}\text { Arm amputation; Drug } \\
\text { dependence (including } \\
\text { cannabis, } \\
\text { benzodiazepines and } \\
\text { heroin); Limb } \\
\text { reduction defect; } \\
\text { Alcohol use }\end{array}$ & $\begin{array}{c}\text { Drug abuse; Loss of consciousness; } \\
\text { Hypothermia }\end{array}$ \\
\hline 30 & M, Adult (31yy) & N/A & & & $\begin{array}{c}\text { Abnormal behavior; Myocardial } \\
\text { infarction; } \\
\text { Drug abuse (heroin, cocaine); } \\
\text { Psychomotor hyperactivity; } \\
\text { Peripheral coldness; } \\
\text { Unresponsive to stimuli; } \\
\text { Arteriosclerosis coronary artery; Blood } \\
\text { glucose decreased }\end{array}$ \\
\hline 31. & F, Adult (72yy) & $200 \mathrm{mg}$ & Opioids & $\begin{array}{c}\text { Schizophrenia; } \\
\text { Depression; } \\
\text { Somnolence; Anxiety }\end{array}$ & $\begin{array}{l}\text { Movement disorder; Sudden death; Drug } \\
\text { abuse }\end{array}$ \\
\hline 32. & M, Adult (27yy) & $400 \mathrm{mg}$ & Antipsychotics & $\begin{array}{l}\text { Asthma; Nicotine } \\
\text { dependence; Drug } \\
\text { abuse; Obesity; } \\
\text { Substance use; } \\
\text { Diabetes mellitus; } \\
\text { Mental disorder; } \\
\text { Alcohol use }\end{array}$ & Asphyxia; Substance abuse \\
\hline 33. & $\begin{array}{l}\text { M, Adult (50 } \\
\text { yy) }\end{array}$ & $550 \mathrm{mg}$ & & & $\begin{array}{c}\text { Hypoxic-ischemic encephalopathy; Drug } \\
\text { abuse (cocaine); Pulmonary } \\
\text { hemosiderosis; Pulmonary edema; } \\
\text { Cardiac arrest; } \\
\text { Left ventricular hypertrophy; } \\
\text { Arteriosclerosis coronary artery; Aortic } \\
\text { arteriosclerosis; } \\
\text { Myocardial fibrosis; Hepatic steatosis; } \\
\text { Spleen congestion }\end{array}$ \\
\hline 34. & M, Adult (29yy) & $1000 \mathrm{mg}$ & Opioids & Schizophrenia & Substance abuse (heroin) \\
\hline 35. & F, Adult & N/A & Antidepressants & & Drug abuse \\
\hline 36. & M, Adult (34yy) & $700 \mathrm{mg}$ & & $\begin{array}{l}\text { Schizophrenia; } \\
\text { Nicotine dependence; } \\
\text { Drug abuse }\end{array}$ & Cardiac arrest; Substance abuse \\
\hline 37. & F, Adult (28yy) & N/A & Antidepressants & & Drug abuse \\
\hline 38. & M & $100 \mathrm{mg}$ & & $\begin{array}{c}\text { Blood cholesterol } \\
\text { increased; Bipolar } \\
\text { disorder; } \\
\text { Gastroesophageal } \\
\text { reflux; Schizoaffective } \\
\text { disorder; Diabetes } \\
\text { mellitus }\end{array}$ & $\begin{array}{l}\text { Sudden death; Drug abuse (cocaine and } \\
\text { another illicit drug) }\end{array}$ \\
\hline 39. & F, Adult (50yy) & N/A & Alcohol & $\begin{array}{l}\text { Drug abuse; Drug } \\
\text { dependence }\end{array}$ & $\begin{array}{l}\text { Completed suicide; Toxicity to various } \\
\text { agents; Drug abuse (propofol) }\end{array}$ \\
\hline 40 & M & N/A & & $\begin{array}{l}\text { Product use issue; } \\
\text { Psychotic disorder }\end{array}$ & $\begin{array}{l}\text { Product use issue; Drug level increased } \\
\text { (clozapine levels in the } 600,000 \text { s); Death; } \\
\text { Adverse event; Psychotic disorder; } \\
\text { Intentional product misuse }\end{array}$ \\
\hline
\end{tabular}


Table 4. Cont.

\begin{tabular}{|c|c|c|c|c|c|}
\hline & & $\begin{array}{c}\text { Clozapine } \\
\text { Dosage (mg) }\end{array}$ & $\begin{array}{l}\text { Concomitant } \\
\text { Drugs }\end{array}$ & Medical History & $\begin{array}{l}\text { Reactions according to the MedDRA } \\
\text { Dictionary (Preferred Terms-PT) }\end{array}$ \\
\hline 41. & M & N/A & & & $\begin{array}{c}\text { Drug withdrawal syndrome; Memory } \\
\text { impairment; Disorientation; } \\
\text { Completed suicide }\end{array}$ \\
\hline 42. & F, Adult (28yy) & N/A & Antidepressants & & Toxicity to various agents; Drug abuse \\
\hline 43. & M, Adult (31yy) & $700 \mathrm{mg}$ & & $\begin{array}{l}\text { Anxiety; Psychotic } \\
\text { disorder; Depression }\end{array}$ & $\begin{array}{c}\text { Completed suicide (suicide due to } \\
\text { overdose on clozapine); } \\
\text { Intentional product misuse; } \\
\text { Overdose; Adverse event }\end{array}$ \\
\hline 44. & M & N/A & & & $\begin{array}{l}\text { Completed suicide (patient jumped to his } \\
\text { death); Withdrawal syndrome } \\
\text { (periodically he would refrain from } \\
\text { taking the medication, thus suffering } \\
\text { withdrawal/Initially withdrawal } \\
\text { symptoms would consist of } \\
\text { disorientation after 2-3 days and memory } \\
\text { impairment after } 4-7 \text { days) }\end{array}$ \\
\hline 45. & M & N/A & & & $\begin{array}{c}\text { Death; Withdrawal syndrome } \\
\text { (withdrawal symptoms from the capsule } \\
\text { form of Clozaril antipsychotic } \\
\text { medication) }\end{array}$ \\
\hline 46. & M, Adult (58yy) & $100 \mathrm{mg}$ & & $\begin{array}{l}\text { Alcohol use; } \\
\text { Hypertension }\end{array}$ & $\begin{array}{c}\text { Drug abuse; Vomiting; } \\
\text { Asphyxia; Prescription drug used } \\
\text { without a prescription (abuse of } \\
\text { clozapine } 100 \mathrm{mg} \text { tablets) }\end{array}$ \\
\hline
\end{tabular}

In a number of cases, reporters had omitted some relevant details, including diagnosis, drugs involved, and, more importantly, both the white blood cell (WBC) and the absolute neutrophil count (ANC) results. Out of a total of 599 ADRs, referring to 559 individual cases, some 149 ADRs (42 individual cases) mentioned that the WBC count had been carried out. However, the results were reported in only 123 ADRs (32 individual cases). For 68 of these ADRs, the WBC/ANC count was within the normal range; while 42 and 13 ADRs were, respectively, associated with either leukopenia or increased WBC count levels. However, since modifications overtime of WBC count levels were not reported, it would be problematic to unequivocally conclude from here if clozapine had been stopped in association with the occurrence of WBC count abnormalities.

A total of 28 cases reported here catatonia, six of which were specifically described as being associated with clozapine withdrawal issues; further reporters' comments relating to overdose, suicidal behavior, withdrawal, and abuse issues are listed in Table 5. 
Table 5. Reporter' comments recorded in the line listing; EudraVigilance (EV) clozapine-related misuse/abuse/dependence and withdrawal ADRs (2005-June 2018).

\begin{tabular}{|c|c|}
\hline & Comments in the ADRs Reported in the Dataset \\
\hline $\begin{array}{l}\text { Overdose and suicidal } \\
\text { behaviour ADRs }\end{array}$ & $\begin{array}{l}\text { 1. "Clozapine overdose in suicidal attempt" } \\
\text { 2. "Patient stopped treatment and then took an overdose of about } \\
\text { 3. "Clozapine levels in the } 600,000 \text { 's possible overdose" } \\
\text { 4. "Clozapine overdose of } 3000 \mathrm{mg} / \text { took two weeks supply of } \\
\text { 5. "Clozaril as } 4200 \mathrm{mg}(300 \mathrm{mg} \times 14 \mathrm{dd}) \text { " } \\
\text { 6. "Took an overdose of } 5600 \mathrm{mg} \text { " } \\
\text { 7. "The patient took } 1300 \mathrm{mg} \text { for intentional self-injury" } \\
\text { 8. "Suicide due to overdose on clozapine" }\end{array}$ \\
\hline $\begin{array}{l}\text { Withdrawal and clozapine } \\
\text { discontinuation ADRs }\end{array}$ & $\begin{array}{l}\text { 1. "Clozaril withdrawal due to non-compliance" } \\
\text { 2. "Withdrawal reaction cholinergic rebound" } \\
\text { 3. "Anticholinergic withdrawal syndrome" } \\
\text { 4. "Withdrawal psychotic episode" } \\
\text { 5. "Sudden withdrawal with Clozaril" } \\
\text { 6. "Clozaril withdrawal psychosis" } \\
\text { 7. "Psychotic decompensation after clozapine discontinuation" } \\
\text { 8. "Relapse psychosis" } \\
\text { 9. "Experiencing extremely negative reactions due to going off } \\
\text { 10. "Serotonin syndrome associated with clozapine withdrawal" } \\
\text { 11. "Withdrawal agitation" } \\
\text { 12. "Tachycardia and high BP possibly due to Clozaril withdrawal" } \\
\text { 13. "Acute clozapine withdrawal" } \\
\text { 14. "Withdrawal symptoms from Clozaril" } \\
\text { 15. "Compatible with withdrawal effects arising from the abrupt }\end{array}$ \\
\hline Abuse of clozapine & $\begin{array}{l}\text { 1. "Abusing Clozaril taking more than he needs" } \\
\text { 2. "Clozaril was prescribed for family members" } \\
\text { 3. "Took his wife's Clozaril to help him sleep" } \\
\text { 4. "He depended on clozapine" } \\
\text { 5. "Died because of clozapine and alcohol" } \\
\text { 6. "Clozapine as a drug of abuse" } \\
\text { 7. "Clozaril misuse" } \\
\text { 8. "Abuse of Leponex" } \\
\text { 9. "Patient possibly has used Leponex as drug of abuse or he bought } \\
\end{array}$ \\
\hline
\end{tabular}

\section{Discussion}

To the best of our knowledge, this is the largest collection of literature data relating to clozapine withdrawal and misuse/abuse/dependence cases. Our analysis found 599 ADRs of interest (relating to 559 individuals), representing 5.05\% of all 11,847 reports submitted to the EMA EV between 2005 and 2018 and judged as 'suspect.' In contrast with the related knowledge on this topic, mostly focused on small case reports/series [10,43-48], current findings referred to high numbers of patients presenting with clozapine withdrawal and misusing issues.

Clozapine ADRs of interest showed a peak in 2008, but an overall increase was observed between 2010 and 2018. One could argue that this upward trend may have been associated with a range of factors, including the increasing rates of worldwide availability of clozapine due to an overwhelming 
evidence of its effectiveness [2,49-51] and/or a major awareness of the relevant and significant impact of pharmacovigilance processes and issues, resulting in a rise of spontaneous reporting practices related to safety monitoring of medicinal products in general [52].

Withdrawal cases collected here were recorded according to the related PTs defined in the MedDRA dictionary [35], where withdrawal is described as "the abrupt cessation of a drug use in a habituated person; and a substance specific syndrome following cessation or reduction in intake of a psychoactive substance previously used regularly". Clozapine withdrawal/discontinuation ADRs were the most frequently reported and, as such, current findings confirmed and expanded on previous anecdotal data $[6,53,54]$. In association with a sudden discontinuation of clozapine, which may be required in cases of blood dyscrasia or suspected myocarditis, instances of withdrawal have already been reported $[6,44,54,55]$. It is of clear interest that a case of clozapine withdrawal neonatal syndrome was specifically mentioned here as well. The clozapine multi-receptor agonism/antagonism is likely responsible for the occurrence of discontinuation/withdrawal symptoms. Indeed, the clozapine pharmacodynamic profile may well include: (a) a dopaminergic super-sensitivity, with the risk of a dopaminergic psychosis and symptoms such as dystonias, dyskinesias, and catatonia [38,56-59]; (b) a cholinergic rebound, inducing in vulnerable patients a rapid worsening of psychosis, agitation, confusion, insomnia, and symptoms including nausea, vomiting, diarrhea, headache, diaphoresis, and abnormal movements, such as dystonias and dyskinesias [6,54,56,57,60-64]. Consistent with this, symptoms appear to regress rapidly with the help of anti-cholinergic drugs; (c) a serotonergic syndrome, which may occur even without the concomitant use of a serotonergic agent $[10,39,61]$. In fact, acting as a 5-HT2A antagonist, long-term clozapine use may be associated with receptor downregulation, and thus, its abrupt discontinuation might lead to receptors' upregulation $[65,66]$; (d) a sudden decrease in gamma-aminobutyric acid (GABA) activity, with the development of catatonic symptoms which may include, mutism, waxy flexibility, staring, posturing, mannerisms, negativism, and also restless, irrelevant speech, and psychomotor agitation [6,67]. The clozapine agonist action on GABA receptors can explain both the drug-drug interaction between clozapine and benzodiazepines, and the flumazenil therapeutic effect in clozapine intoxication cases [6]; and (e) a modification of norepinephrine levels, with clozapine abrupt discontinuation in chronic patients possibly resulting in an increase in suicidal behavior [1,68]. As it may occur with remaining psychotropics, such as antidepressants $[69,70]$, the existence of a discontinuation syndrome following an abrupt stoppage of, or marked reduction in, the dosage of a drug taken on a regular basis does not necessarily mean that a drug causes dependence. Discontinuation should be seen here as distinct from the withdrawal scenario associated with alcohol and other addictive substances, a scenario which commonly presents together with craving, drug seeking behavior, and the inability to stop drug use [71,72]. Thus, if a discontinuation of clozapine is needed, the molecule should be gradually tapered off over several weeks rather than abruptly discontinued, except in cases of emergency (e.g., agranulocytosis), and only with close clinical monitoring $[10,67,73]$. There are no established guidelines regarding which antipsychotic to choose after withdrawal of clozapine [73], although anticholinergics and olanzapine may be the treatment of choice for preventing withdrawal [53].

The reporters' narrative here formally submitted in a few cases was consistent with both drug seeking and diversion behavior (e.g., " ... clozapine as a drug of abuse ... "; and " .. patient possibly has used Leponex as drug of abuse or he bought Leponex for drug abuse by others ... ".). Considering the current misuse/abuse issues, the number of clozapine related ADRs (e.g., 326 ADRs; referring to: 'drug abuse', 'drug abuser', 'drug diversion', 'intentional product misuse', 'product use issue', and 'substance abuse') here identified may be difficult to interpret. In fact, in comparing quetiapine- and other second-generation antipsychotic-related intentional abuse exposures reported to the US National Poison Data System, Klein et al. [74] suggested that clozapine and olanzapine were significantly associated with frequent instances of severe central effects, including: lethargy/drowsiness/slurred speech; agitation/irritability; confusion and hallucinations. However, no use of clozapine as a recreational drug was reported. One could argue that at least a proportion of 
these ADRs involved subjects suffering from both schizophrenia and a co-occurring SUD. Furthermore, current findings here did not identify any idiosyncratic intake modalities (e.g., intravenous use) that are typical of a substance misuse behavior. Hypothetically, putative levels of clozapine misuse liability might be tentatively explained considering the range of its pharmacodynamics activities, and the occurrence of rewarding and pleasurable effects due to the agonism at both delta-opioid $[13,17,75]$ and cannabinoid CB1 receptors [18]; and the antagonism at muscarinic receptors [76,77]. Additionally, although clozapine was here ingested on its own in some $69 \%$ of cases, remaining antipsychotics and benzodiazepines were the drugs most frequently reported in association. Polypharmacy ingestion may have facilitated the occurrence of synergistic reactions, and hence the EMA ADRs' reporting, due to possible increase in clozapine plasma concentrations associated with metabolism inhibition [14]. Relatively small recreational drugs' numbers were identified here as well, and this may have been associated with complex pharmacological interactions. Indeed, clozapine pre-treatment may increase cocaine concentrations, but significantly reduce subjective responses to cocaine [78].

Regarding the ADRs' outcomes, present figures seem to be a reason for concern, since most cases $(298 / 559=53.3 \%)$ required a prolonged hospitalization. Furthermore, some 46 ( $8.2 \%$ of 559 subjects $)$ fatalities were here reported, and these mostly occurred in the context of: high dosage clozapine intake; suicidal behavior; and/or polydrug abuse. Although the EMA suggests a maximum clozapine dosage of up to $900 \mathrm{mg} /$ day and warns about side-effects occurring at doses over $450 \mathrm{mg} /$ day [79], cases reported here were at times associated with massive dosages (e.g., in the range of 2800-5600 mg), although most of these cases were associated with overdose/suicide instances. These rates are a reason for concern but seem to be consistent with the suggestion that those subjects diagnosed with treatment-resistant schizophrenia, and hence, who are typically being prescribed with clozapine, present with a higher risk of attempted/completed suicide in comparison to the general population [80]. Clozapine overdose may be lethal due to changes in heart rhythm, respiratory depression, and altered state of consciousness $[4,81,82]$; clozapine high-dosage ingestion may be associated with a mortality rate of approximately $12 \%$ [83], with fatalities most typically being associated with cardiac insufficiency and aspiration pneumonia observed with dosages higher than $2 \mathrm{~g}$ [45-48]. Although cases of full recovery after ingestion of either high dosages [46] and/or associated with very high plasma levels (>9000 ng/mL) [45] have been reported, ingestion of $400 \mathrm{mg}$ in a patient not previously treated may be life-threatening [46].

\subsection{Limitations}

Overall, pharmacovigilance systems such as the EV in Europe, the Medicines and Healthcare products Regulatory Agency (MHRA) in the United Kingdom and the FDA Adverse Event Reporting System (FAERS) are considered important because the analysis of suspected ADRs may deal with early detection of possible drug safety signals [34]. However, the study of ADRs alone may not be enough to prove causality between a certain suspected reaction and a specific medicine. Any case report should be considered and assessed together with all available data, including case reports worldwide, clinical trials, epidemiological studies, and toxicological investigations.

Unfortunately, the dataset did not typically report here any clinical data, including information on the current/past psychopathology and the medications that were prescribed in association. Furthermore, the study of ADRs may be influenced by the molecule's availability and extent of use, although precise data on the worldwide prescription rates for clozapine were not here identified or available. Moreover, in being voluntarily reported/submitted, the number of ADRs could have been conditioned by both the nature of the reaction and the public awareness of a safety concern, with underreporting possibly having here occurred. Additionally, some data could have been unavailable, invalid or redacted.

\subsection{Conclusions}

Current findings focused on a range of clozapine withdrawal and misuse/abuse/dependence issues. It was here confirmed, on a large-scale basis, that, following the abrupt reduction of clozapine 
dosage a discontinuation/withdrawal syndrome may occur. This may involve possible severe and long-lasting symptoms $[10,59,67,84]$. Moreover, although this may be an unusual event, it is here suggested that cases of misuse/abuse/dependence could occur in patients with a concomitant SUD and this is despite clozapine being of clinical value for the treatment of dual diagnosis. Conversely, current findings emphasized a range of severe and fatal health consequences associated with clozapine high-dosage intake, and patients taking clozapine should be educated thoroughly about the risks associated with polydrug intake. In being a prescribed drug of highly significant clinical value [85], increased awareness is essential towards prevention, diagnosis, and treatment of cases of clozapine abuse/recreational use in patients diagnosed with schizophrenia [86].

Author Contributions: S.C. conceived the paper, the main conceptual ideas, proof outline and designed the study. S.C. wrote the first draft and led on the analysis of the results with the support of A.G. and J.M.C. under the supervision of F.S. All authors have read and agreed to the published version of the manuscript.

Funding: This research received no external funding.

Conflicts of Interest: F.S. was a member of the UK Advisory Council on the Misuse Drugs (ACMD) from 2013 to 2019; he is currently an EMA Advisory Board (psychiatry) member. J.M.C. is a member of the ACMD's Novel Psychoactive Substances and Technical Committees. The authors have no other relevant affiliations or financial involvement with any organization or entity with a financial interest in or financial conflict with the subject matter or materials discussed in the manuscript.

\section{References}

1. Khokhar, J.; Henricks, A.M.; Sullivan, E.D.; Green, A.I. Unique Effects of Clozapine: A Pharmacological Perspective. Adv. Pharmacol. 2018, 82, 137-162.

2. Warnez, S.; Alessi-Severini, S. Clozapine: A review of clinical practice guidelines and prescribing trends. BMC Psychiatry 2014, 14, 102. [CrossRef]

3. ArrayExpress-A database of functional genomics experiments. Available online: http://www.ebi.ac.uk/ arrayexpress/ (accessed on 12 November 2012).

4. Stahl, S.M. Stahl's Essential Psychopharmacology-Prescriber's Guide, 6th ed.; Cambridge University Press: Cambridge, UK, 2017.

5. Mauri, M.C.; Paletta, S.; Maffini, M.; Colasanti, A.; Dragogna, F.; Di Pace, C.; Altamura, A. Clinical pharmacology of atypical antipsychotics: An update. EXCLI J. 2014, 13, 1163-1191.

6. Lawrence, R.E.; First, M.B.; Lieberman, J.A. Schizophrenia and Other Psychoses; Wiley: Hoboken, NJ, USA, 2015; Volume 1, pp. 791-856.

7. O Kalkman, H.; Loetscher, E. alpha2C-Adrenoceptor blockade by clozapine and other antipsychotic drugs. Eur. J. Pharmacol. 2003, 462, 33-40. [CrossRef]

8. Fjalland, B.; Boeck, V. Neuroleptic Blockade of the Effect of Various Neurotransmitter Substances. Acta Pharmacol. Toxicol. 1978, 42, 206-211. [CrossRef] [PubMed]

9. Bilbily, J.; Mccollum, B.; De Leon, J. Catatonia Secondary to Sudden Clozapine Withdrawal: A Case with Three Repeated Episodes and a Literature Review. Case Rep. Psychiatry 2017, 2017, 1-11. [CrossRef] [PubMed]

10. Stevenson, E.; Schembri, F.; Green, D.M.; Burns, J. Serotonin Syndrome Associated with Clozapine Withdrawal. JAMA Neurol. 2013, 70, 1054. [CrossRef]

11. Meltzer, H.Y. An overview of the mechanism of action of clozapine. J. Clin. Psychiatry 1994, 55.

12. Schwieler, L.; Engberg, G.; Erhardt, S. Clozapine modulates midbrain dopamine neuron firing via interaction with the NMDA receptor complex. Synapse 2004, 52, 114-122. [CrossRef]

13. Kobayashi, T.; Ikeda, K.; Kumanishi, T. Effects of clozapine on the delta- and kappa- opioid receptors and the G-protein-activated K+ (GIRK) channel expressed in Xenopus oocytes. Br. J. Pharmacol. 1998, 123, 421-426. [CrossRef]

14. Aronson, J.K. Clozapine. In Meyler's Side Effects of Drugs (Sixteenth Edition): The International Encyclopedia of Adverse Drug Reactions and Interactions; Elsevier: Kidlington, UK, 2016; pp. 443-477.

15. Trenton, A.J.; Currier, G.W.; Zwemer, F.L. Fatalities associated with therapeutic use and overdose of atypical antipsychotics. CNS Drugs 2003, 17, 307-324. [CrossRef] [PubMed]

16. Khokhar, J.; Dwiel, L.L.; Henricks, A.M.; Doucette, W.T.; Green, A.I. The link between schizophrenia and substance use disorder: A unifying hypothesis. Schizophr. Res. 2018, 194, 78-85. [CrossRef] [PubMed] 
17. Olianas, M.C.; Dedoni, S.; Ambu, R.; Onali, P.L. Agonist activity of N-desmethylclozapine at $\delta$-opioid receptors of human frontal cortex. Eur. J. Pharmacol. 2009, 607, 96-101. [CrossRef] [PubMed]

18. Sundram, S.; Copolov, D.; Dean, B. Clozapine decreases [3H] CP 55940 binding to the cannabinoid1 receptor in the rat nucleus accumbens. Naunyn-Schmiedeberg's Arch. Pharmacol. 2005, 371, 428-433. [CrossRef]

19. Chung, P.C.S.; Kieffer, B.L. Delta opioid receptors in brain function and diseases. Pharmacol. Ther. 2013, 140, 112-120. [CrossRef]

20. Schifano, F.; Chiappini, S.; Corkery, J.M.; Guirguis, A. Abuse of Prescription Drugs in the Context of Novel Psychoactive Substances (NPS): A Systematic Review. Brain Sci. 2018, 8, 73. [CrossRef]

21. Bersani, F.S.; Corazza, O.; Simonato, P.; Mylokosta, A.; Levari, E.; Lovaste, R.; Schifano, F. Drops of madness? Recreational misuse of tropicamide collyrium; early warning alerts from Russia and Italy. Gen. Hosp. Psychiatry 2013, 35, 571-573. [CrossRef]

22. Wu, Y.; Blichowski, M.; Daskalakis, Z.J.; Wu, Z.; Liu, C.C.; Cortez, M.A.; Snead, O.C. Evidence that clozapine directly interacts on the GABAB receptor. NeuroReport 2011, 22, 637-641. [CrossRef]

23. López-Gil, X.; Artigas, F.; Adell, A. Unraveling monoamine receptors involved in the action of typical and atypical antipsychotics on glutamatergic and serotonergic transmission in prefrontal cortex. Curr. Pharm. Des. 2010, 16, 502-515. [CrossRef]

24. Schifano, F.; Orsolini, L.; Papanti, G.D.; Corkery, J.M. Novel psychoactive substances of interest for psychiatry. World Psychiatry 2015, 14, 15-26. [CrossRef]

25. Schifano, F.; Napoletano, F.; Arillotta, D.; Zangani, C.; Gilgar, L.; Guirguis, A.; Corkery, J.M.; Vento, A. The clinical challenges of synthetic cathinones. Br. J. Clin. Pharmacol. 2019. [CrossRef] [PubMed]

26. Zimmet, S.V.; Strous, R.D.; Burgess, E.S.; Kohnstamm, S.; Green, A.I. Effects of clozapine on substance use in patients with schizophrenia and schizoaffective disorder: A retrospective survey. J. Clin. Psychopharmacol. 2000, 20, 94-98. [CrossRef] [PubMed]

27. Krause, M.; Huhn, M.; Schneider-Thoma, J.; Bighelli, I.; Gutsmiedl, K.; Leucht, S. Efficacy, acceptability and tolerability of antipsychotics in patients with schizophrenia and comorbid substance use. A systematic review and meta-analysis. Eur. Neuropsychopharmacol. 2019, 29, 32-45. [CrossRef] [PubMed]

28. Brunette, M.F.; Drake, R.E.; Xie, H.; McHugo, G.J.; Green, A.I. Clozapine Use and Relapses of Substance Use Disorder Among Patients with Co-occurring Schizophrenia and Substance Use Disorders. Schizophr. Bull. 2006, 32, 637-643. [CrossRef]

29. Okhuijsen-Pfeifer, C.; Sterk, A.Y.; Horn, I.M.; Terstappen, J.; Kahn, R.S.; Luykx, J.J. Demographic and clinical features as predictors of clozapine response in patients with schizophrenia spectrum disorders: A systematic review and meta-analysis. Neurosci. Biobehav. Rev. 2020, 111, 246-252. [CrossRef]

30. Chang, J.; Kim, S.; Lee, G.; Kim, E.; Jung, H. Quetiapine misuse and abuse: Is it an atypical paradigm of drug seeking behavior? J. Res. Pharm. Pr. 2017, 6, 12-15. [CrossRef]

31. Chiappini, S.; Schifano, F. Is There a Potential of Misuse for Quetiapine? Literature Review and Analysis of the European Medicines Agency/European Medicines Agency Adverse Drug Reactions' Database. J. Clin. Psychopharmacol. 2018, 38, 72-79. [CrossRef]

32. Evoy, K.; Teng, C.; Encarnacion, V.G.; Frescas, B.; Hakim, J.; Saklad, S.; Frei, C.R. Comparison of Quetiapine Abuse and Misuse Reports to the FDA Adverse Event Reporting System with Other Second-Generation Antipsychotics. Subst. Abus. Res. Treat. 2019, 13. [CrossRef]

33. Vento, A.E.; Kotzalidis, G.D.; Cacciotti, M.; Papanti, G.D.; Orsolini, L.; Rapinesi, C.; Savoja, V.; Calabrò, G.; Del Casale, A.; Piacentino, D.; et al. Quetiapine Abuse Fourteen Years Later: Where Are We Now? A Systematic Review. Subst. Use Misuse 2019, 55, 304-313. [CrossRef]

34. European Medicines Agency (EMA). Guideline on good pharmacovigilance practices, Module VI-Collection, management and submission of reports of suspected adverse reactions to medicinal products (Rev 2). Available online: http://www.ema.europa.eu/docs/en_GB/document_library/Regulatory_and_procedural_ guideline/2017/08/WC500232767.pdf (accessed on 25 July 2019).

35. MedDRA. Version 21. 2018. Available online: https://www.meddra.org/sites/default/files/guidance/file/smq intguide_21_0_english.pdf (accessed on 25 July 2019).

36. Schifano, F.; Chiappini, S.; Corkery, J.M.; Guirguis, A. Assessing the 2004-2018 Fentanyl Misusing Issues Reported to an International Range of Adverse Reporting Systems. Front. Pharmacol. 2019, 10, 46. [CrossRef] 
37. Schifano, F.; Chiappini, S.; Corkery, J.M.; Guirguis, A. An Insight into Z-Drug Abuse and Dependence: An Examination of Reports to the European Medicines Agency Database of Suspected Adverse Drug Reactions. Int. J. Neuropsychopharmacol. 2019, 22, 270-277. [CrossRef] [PubMed]

38. Schifano, F.; Chiappini, S. Is there such a thing as a 'lope' dope? Analysis of loperamide-related European Medicines Agency (EMA) pharmacovigilance database reports. PLoS ONE 2018, 13, e0204443. [CrossRef] [PubMed]

39. Schifano, F.; Chiappini, S. Is There a Potential of Misuse for Venlafaxine and Bupropion? Front. Pharmacol. 2018, 9. [CrossRef] [PubMed]

40. European Medicines Agency (EMA). Note for Guidance-EudraVigilance Human-Processing of Safety Messages and Individual Case Safety Reports (ICSRs) (EMA/H/20665/04/Final Rev. 2). Available online: https://www.ema.europa.eu/en/documents/regulatory-procedural-guideline/note-guidanceeudravigilance-human-processing-safety-messages-individual-case-safety-reports-icsrs_en.pdf (accessed on 25 July 2019).

41. European Medicines Agency (EMA). EudraVigilance Working Group (EV-EWG). Guideline on the Use of Statistical Signal Detection Methods in the EudraVigilance Data Analysis System. Available online: https://www.ema.europa.eu/en/documents/regulatory-procedural-guideline/draft-guideline-usestatistical-signal-detection-methods-eudravigilance-data-analysis-system_en.pdf (accessed on 25 July 2019).

42. European Medicines Agency (EMA). ICH Guideline E2B (R3) on Electronic Transmission of Individual Case Safety Reports (ICSRs)—Data Elements and Message Specification-Implementation Guide. Available online: https://www.ema.europa.eu/en/documents/scientific-guideline/international-conferenceharmonisation-technical-requirements-registration-pharmaceuticals-human-use_en-4.pdf (accessed on 25 July 2019).

43. Bastiampillai, T.; Forooziya, F.; Dhillon, R. Clozapine-withdrawal catatonia. Aust. N. Z. J. Psychiatry 2013, 43, 283-284.

44. Goudie, A.J.; Smith, J.A.; Robertson, A.; Cavanagh, C. Clozapine as a drug of dependence. Psychopharmacology 1999, 142, 369-374. [CrossRef]

45. Ines, S.; Mahal, A.; Hoss, J.; Gaertner, H.J. Intoxication with clozapine: Plasma levels above $9000 \mathrm{ng} / \mathrm{mL}$. Typical clinical picture diagnostic confusion (abstract). Neuropsychopharmacology 1994, 10, $122 \mathrm{~S}$.

46. Le Blaye, I.; Donatini, B.; Hall, M. Acute over dosage with clozapine: A review of the available clinical experience. Pharm. Med. 1992, 6, 169-178.

47. Piccini, G.; Ceroni, P.; Marchesi, C.; Maggini, C.; Maestri, G. Acute clozapine overdosage. Br. J. Psychiatry 1997, 170, 290. [CrossRef]

48. Worm, K.; Kringsholm, B.; Steentoft, A. Clozapine cases with fatal, toxic or therapeutic concentrations. Int. J. Leg. Med. 1993, 106, 115-118. [CrossRef]

49. Niehues, G.D.; Balan, A.B.; Prá, V.B.; Pellizzaro, R.S.; Da Silva, P.R.A.; Schwarzbold, M.L.; Diaz, A.P.; Costa, A.P. Trends in the prescription of clozapine in a psychiatric hospital: A 5-year observational study. Trends Psychiatry Psychother. 2017, 39, 158-164. [CrossRef]

50. Nielsen, J.; Røge, R.; Schjerning, O.; Sørensen, H.J.; Taylor, D. Geographical and temporal variations in clozapine prescription for schizophrenia. Eur. Neuropsychopharmacol. 2012, 22, 818-824. [CrossRef] [PubMed]

51. Verdoux, H.; Pambrun, E.; Cortaredona, S.; Coldefy, M.; Le Neindre, C.; Tournier, M.; Verger, P. Geographical disparities in prescription practices of lithium and clozapine: A community-based study. Acta Psychiatr. Scand. 2016, 133, 470-480. [CrossRef] [PubMed]

52. Santoro, A.; Genov, G.; Spooner, A.; Raine, J.; Arlett, P. Promoting and Protecting Public Health: How the European Union Pharmacovigilance System Works. Drug Saf. 2017, 40, 855-869. [CrossRef] [PubMed]

53. Galova, A.; Berney, P.; Desmeules, J.; Sergentanis, I.; Besson, M. A case report of cholinergic rebound syndrome following abrupt low-dose clozapine discontinuation in a patient with type I bipolar affective disorder. BMC Psychiatry 2019, 19, 73. [CrossRef] [PubMed]

54. Lander, M.; Bastiampillai, T.; Sareen, J. Review of withdrawal catatonia: What does this reveal about clozapine? Transl. Psychiatry 2018, 8, 139. [CrossRef] [PubMed]

55. Koychev, I.; Hadjiphilippou, S.; Lynch, J.; Whelan, P.; MacCabe, J. Sudden-Onset Catatonia Following Clozapine Withdrawal: A Case Report. J. Clin. Psychiatry 2016, 77, e899. [CrossRef] 
56. Moncrieff, J. Does antipsychotic withdrawal provoke psychosis? Review of the literature on rapid onset psychosis (supersensitivity psychosis) and withdrawal-related relapse. Acta Psychiatr. Scand. 2006, 114, 3-13. [CrossRef]

57. Sarma, S.; Chetia, D.; Raha, B.; Agarwal, G. Clozapine withdrawal emergent dystonia, oculogyric crisis and rebound psychosis in a single patient. Ther. Adv. Psychopharmacol. 2015, 6, 145-146. [CrossRef]

58. Shore, D.; Matthews, S.; Cott, J.; Lieberman, J.A. Clinical implications of clozapine discontinuation: Report of a NIMH workshop. Schizophr Bull. 1995, 21, 333-338. [CrossRef]

59. Wang, B.Z.; Gupta, A.; Bastiampillai, T.; Sani, F. Recurrent clozapine and lorazepam withdrawal psychosis with catatonia. Aust. New Zealand J. Psychiatry 2012, 46, 795-796. [CrossRef]

60. Breen, E.G. Clozapine withdrawal syndrome. BJPsych Bull. 2017, 41, 366. [CrossRef] [PubMed]

61. Kumar, S.; Sur, S.; Singh, A. Catatonia Following Abrupt Stoppage of Clozapine. Aust. New Zealand J. Psychiatry 2011, 45, 499. [CrossRef] [PubMed]

62. Shiovitz, T.M.; Welke, T.L.; Tigel, P.D.; Anand, R.; Hartman, R.D.; Sramek, J.J.; Kurtz, N.M.; Cutler, N.R. Cholinergic Rebound and Rapid Onset Psychosis Following Abrupt Clozapine Withdrawal. Schizophr. Bull. 1996, 22, 591-595. [CrossRef]

63. Yeh, A.W.-C.; Lee, J.W.Y.; Cheng, T.-C.; Wen, J.-K.; Chen, W.-H. Clozapine withdrawal catatonia associated with cholinergic and serotonergic rebound hyperactivity: A case report. Clin. Neuropharmacol. 2004, 27. [CrossRef] [PubMed]

64. Wadekar, M.; Syed, S. Clozapine-Withdrawal Catatonia. Psychosomatics 2010, 51, 355. [CrossRef]

65. Zerjav-Lacombe, S.; Dewan, V. Possible serotonin syndrome associated with clomipramine after withdrawal of clozapine. Ann. Pharmacother. 2001, 35. [CrossRef] [PubMed]

66. Huang, X.F.; Tan, Y.Y.; Huang, X.; Wang, Q. Effect of chronic treatment with clozapine and haloperidol on 5-HT2A and 2C receptor mRNA expression in the rat brain. Neurosci. Res. 2007, 59, 314-321. [CrossRef]

67. Shields, M.K.; Bastiampillai, T.; Mohan, T. Managing clozapine discontinuation-Acute and chronic maintenance strategies. Aust. New Zealand J. Psychiatry 2012, 46, 1104-1105. [CrossRef]

68. Meltzer, H.Y.; Alphs, L.; Green, A.I.; Altamura, A.C.; Anand, R.; Bertoldi, A.; Bourgeois, M.; Chouinard, G.; Islam, M.Z.; Kane, J.; et al. International Suicide Prevention Trial Study Group. Clozapine treatment for suicidality in schizophrenia: International Suicide Prevention Trial (InterSePT). Arch. Gen. Psychiatry. 2003, 60, 82-91. [CrossRef]

69. Fava, G.A.; Gatti, A.; Belaise, C.; Guidi, J.; Offidani, E. Withdrawal Symptoms after Selective Serotonin Reuptake Inhibitor Discontinuation: A Systematic Review. Psychother. Psychosom. 2015, 84, 72-81. [CrossRef]

70. Gabriel, M.; Sharma, V. Antidepressant discontinuation syndrome. CMAJ 2017, 189, E747. [CrossRef] [PubMed]

71. Schatzberg, A.F.; Haddad, P.; Kaplan, E.M.; Lejoyeux, M.; Rosenbaum, J.F.; Young, A.H.; Zajecka, J. Serotonin reuptake inhibitor discontinuation syndrome: A hypothetical definition. Discontinuation Consensus panel. J. Clin. Psychiatry 1997, 58, 5-10. [PubMed]

72. Shelton, R. The nature of the discontinuation syndrome associated with antidepressant drugs. J. Clin. Psychiatry 2006, 67, 3-7. [PubMed]

73. Szafrański, T.; Gmurkowski, K. Clozapine withdrawal. Psychiatr. Pol. 1999, 33, 51-67.

74. Klein, L.; Bangh, S.; Cole, J.B. Intentional Recreational Abuse of Quetiapine Compared to Other Second-generation Antipsychotics. West. J. Emerg. Med. 2016, 18, 243-250. [CrossRef]

75. Pradhan, A.A.; Befort, K.; Nozaki, C.; Gavériaux, C.; Kieffer, B.L. The delta opioid receptor: An evolving target for the treatment of brain disorders. Trends Pharmacol. Sci. 2011, 32, 581-590. [CrossRef]

76. Solismaa, A.; Kampman, O.; Lyytikäinen, L.-P.; Seppälä, N.; Viikki, M.; Mononen, N.; Lehtimaki, T.; Leinonen, E. Genetic Polymorphisms Associated with Constipation and Anticholinergic Symptoms in Patients Receiving Clozapine. J. Clin. Psychopharmacol. 2018, 38, 193-199. [CrossRef]

77. Torrents, R.; Ferré, J.F.; Konareff, A.; Hemery, P.; Sherwin, K.; Lassalle, C.; Simon, N.; Scerra, S. Misuse of Trihexyphenidyl (Artane) on Réunion Island. J. Clin. Psychopharmacol. 2018, 38, 250-253. [CrossRef]

78. Farren, C.; Hameedi, F.A.; Rosen, M.A.; Woods, S.; Jatlow, P.; Kosten, T.R. Significant interaction between clozapine and cocaine in cocaine addicts. Drug Alcohol Depend. 2000, 59, 153-163. [CrossRef] 
79. European Medicines Agency (EMA); Committee for Proprietary Medicinal Products (CPMP). Summary information on referral opinion following arbitration pursuant to Article 30 of Council Directive 2001/83/EC for Leponex and associated names. Available online: https://www.ema.europa.eu/en/medicines/human/ referrals/leponex (accessed on 24 July 2019).

80. Kasckow, J.; Felmet, K.; Zisook, S. Managing suicide risk in patients with schizophrenia. CNS Drugs 2011, 25, 129-143. [CrossRef]

81. He, J.-L.; Xiang, Y.-T.; Li, W.-B.; Cai, Z.-J.; Ungvari, G.S. Hemoperfusion in the Treatment of Acute Clozapine Intoxication in China. J. Clin. Psychopharmacol. 2007, 27, 667-671. [CrossRef] [PubMed]

82. Broich, K.; Heinrich, S.; Marneros, A. Acute Clozapine Overdose: Plasma Concentration and Outcome. Pharmacopsychiatry 1998, 31, 149-151. [CrossRef] [PubMed]

83. Kramer, I.; Rauber-Lüthy, C.; Kupferschmidt, H. Minimal dose for severe poisoning and influence factors in acute human clozapine intoxication: A 13-year retrospective study. Clin. Neuropharm. 2011, 33, 230-234. [CrossRef] [PubMed]

84. Ulrich, S.; Baumann, B.; Wolf, R.; Lehmann, D.; Peters, B.; Bogerts, B.; Meyer, F. Therapeutic drug monitoring of clozapine and relapse-A retrospective study of routine clinical data. Int. J. Clin. Pharmacol. Ther. 2003, 41, 3-13. [CrossRef]

85. Cipriani, A.; Boso, M.; Barbui, C. Clozapine combined with different antipsychotic drugs for treatment resistant schizophrenia. Cochrane Database Syst. Rev. 2009, CD006324.

86. Pandarakalam, J.P. The art of clozapine therapy and "clozaphobia". BMJ 2019, 364, 1484. [CrossRef]

(C) 2020 by the authors. Licensee MDPI, Basel, Switzerland. This article is an open access article distributed under the terms and conditions of the Creative Commons Attribution (CC BY) license (http://creativecommons.org/licenses/by/4.0/). 